\title{
Dual organism design cycle reveals small subunit substitutions that improve [NiFe] hydrogenase hydrogen evolution
}

\author{
Isaac T Yonemoto, Christopher W Matteri, Thao Amy Nguyen, Hamilton O Smith and Philip D Weyman*
}

\begin{abstract}
Background: Photosynthetic microorganisms that directly channel solar energy to the production of molecular hydrogen are a potential future biofuel system. Building such a system requires installation of a hydrogenase in the photosynthetic organism that is both tolerant to oxygen and capable of hydrogen production. Toward this end, we have identified the [NiFe] hydrogenase from the marine bacterium Alteromonas macleodii "Deep ecotype" that is able to be heterologously expressed in cyanobacteria and has tolerance to partial oxygen. The A. macleodii enzyme shares sequence similarity with the uptake hydrogenases that favor hydrogen uptake activity over hydrogen evolution. To improve hydrogen evolution from the A. macleodii hydrogenase, we examined the three Fe-S clusters found in the small subunit of many [NiFe] uptake hydrogenases that presumably act as a molecular wire to guide electrons to or from the active site of the enzyme. Studies by others altering the medial cluster of a Desulfovibrio fructosovorans hydrogenase from $3 \mathrm{Fe}-4 \mathrm{~S}$ to $4 \mathrm{Fe}-4 \mathrm{~S}$ resulted in two-fold improved hydrogen evolution activity.

Results: We adopted a strategy of screening for improved hydrogenase constructs using an Escherichia coli expression system before testing in slower growing cyanobacteria. From the A. macleodii enzyme, we created a mutation in the gene encoding the hydrogenase small subunit that in other systems is known to convert the $3 \mathrm{Fe}-4 \mathrm{~S}$ medial cluster to $4 \mathrm{Fe}-4 \mathrm{~S}$. The medial cluster substitution did not improve the hydrogen evolution activity of our hydrogenase. However, modifying both the medial cluster and the ligation of the distal Fe-S cluster improved in vitro hydrogen evolution activity relative to the wild type hydrogenase by three- to four-fold. Other properties of the enzyme including thermostability and tolerance to partial oxygen did not appear to be affected by the substitutions.
\end{abstract}

Conclusions: Our results show that substitution of amino acids altering the ligation of Fe-S clusters in the $A$. macleodii [NiFe] uptake hydrogenase resulted in increased hydrogen evolution activity. This activity can be recapitulated in multiple host systems and with purified protein. These results validate the approach of using an E. coli-cyanobacteria shuttle system for enzyme expression and improvement.

Keywords: Hydrogenase, Cyanobacteria, Iron-sulfur Cluster, Alteromonas Macleodii "Deep Ecotype"

\section{Background}

Hydrogen produced photobiologically from sunlight and water is a potential future biofuel and is an alternative to traditional carbon-based fuels [1-4]. Enzymes that produce hydrogen include nitrogenases and hydrogenases, the latter comprising the unrelated families of [Fe-Fe] hydrogenases, Fe-only hydrogenases, and [NiFe] hydrogenase enzymes [5]. Cyanobacteria such as Synechococcus elongatus are

\footnotetext{
* Correspondence: pweyman@jcvi.org

J. Craig Venter Institute, 10355 Science Center Dr, San Diego, CA 92121, USA
}

ideal host strains for a biological system that converts solar energy to hydrogen because they are genetically tractable and easily cultured [6]. Because cyanobacteria also perform oxygenic photosynthesis, the oxygen produced will inactivate most hydrogenases $[7,8]$. Therefore, installing a hydrogenase that is less sensitive to oxygen is a primary design constraint of this system. Among the hydrogenase families, oxygen tolerance has been described only among the $[\mathrm{NiFe}]$ hydrogenases, although not all $[\mathrm{NiFe}]$ hydrogenases are oxygen tolerant [9-11]. There is some evidence the native cyanobacterial bidirectional hydrogenase may be 
capable of hydrogen production in the presence of oxygen [12], but the energetic consequences of using NADPH as an electron donor makes it less attractive.

We previously reported the discovery and characterization of a novel hydrogenase isolated from Sargasso Sea environmental DNA with high identity to the stable [NiFe] uptake hydrogenase from Thiocapsa roseopersicina [13]. A closelyrelated sequence was later identified in the Alteromonas macleodii 'Deep ecotype' genome (hereafter referred to as the A. macleodii hydrogenase), and was characterized as active in the presence of $2 \%$ oxygen [14]. The DNA encoding the enzyme was then cloned and used to express active enzyme in both Escherichia coli [15] and S. elongatus [16]. Expressing active $A$. macleodii hydrogenase in both $E$. coli and cyanobacteria requires the co-expression of at least nine additional maturation factors to properly assembly an active enzyme [17]. Due to the complex maturation requirements of [NiFe] hydrogenases, only a few other examples exist of heterologous expression in E. coli [18-20]. The dual expression system is ideal for rapidly generating variant hydrogenases using powerful E. coli genetic tools, quickly screening for desirable properties in $E$. coli, and confirming the persistence of the property in the final target cyanobacterial host.

Although some uptake [NiFe] hydrogenases are oxygentolerant, they are known to be generally directionally biased against hydrogen evolution [21,22]. Enzyme bias in uptake hydrogenases appears to have been altered in separate experiments investigating the ligation of Fe-S clusters in the small subunit of [NiFe] hydrogenases [23,24]. These Fe-S clusters are believed to function as a molecular wire guiding electrons out of the active site and to a biological electron carrier molecule (Figure 1) [10,25,26]. Conversely, in a hydrogen-production scenario, these Fe-S clusters would predominantly operate in the reverse direction, channeling electrons into the active site from an electron donor at the enzyme surface.

At the medial Fe-S cluster, uptake [NiFe] hydrogenases have conserved amino acid residues deviating from the canonical 4-cysteine ligation characteristic of Fe-S cubane clusters. In A. macleodii and almost all members of the uptake NiFe hydrogenase family, the medial cluster features a proline in the place of a ligating cysteine resulting in the installation of a $3 \mathrm{Fe}-4 \mathrm{~S}$ cluster instead of a $4 \mathrm{Fe}-4 \mathrm{~S}$ cluster [26]. Interestingly, the closely related small subunits of the $\mathrm{H}_{2}$-sensing regulatory hydrogenases, which show almost no hydrogen evolution, and the NiFeSe hydrogenases, which show more hydrogen evolution activity [29], both feature cysteinyl ligations at the homologous amino acid position [24,30]. The unrelated small subunit of the F420reducing hydrogenase, which also features $34 \mathrm{Fe}-4 \mathrm{~S}$ clusters, has a $4 \mathrm{Fe}-4 \mathrm{~S}$ cluster for its medial cluster [31]. In $A$. macleodii hydrogenase, and uptake hydrogenases in general, the cluster distal to the active site is a $4 \mathrm{Fe}-4 \mathrm{~S}$ cluster featuring an unusual iron-histidinyl ligation that is conserved in most [NiFe] hydrogenases. While its function is unknown, the unusual ligation may be related to intramolecular electron transfer [23,32].

In Desulfovibrio fructosovorans, substitution of the proline residue associated with the medial cluster to cysteine (Df HynA P238C) resulted in approximately $30 \%$ reduction of hydrogen uptake activity and a nearly two-fold increase in evolution activity [24]. Separately, when the histidine

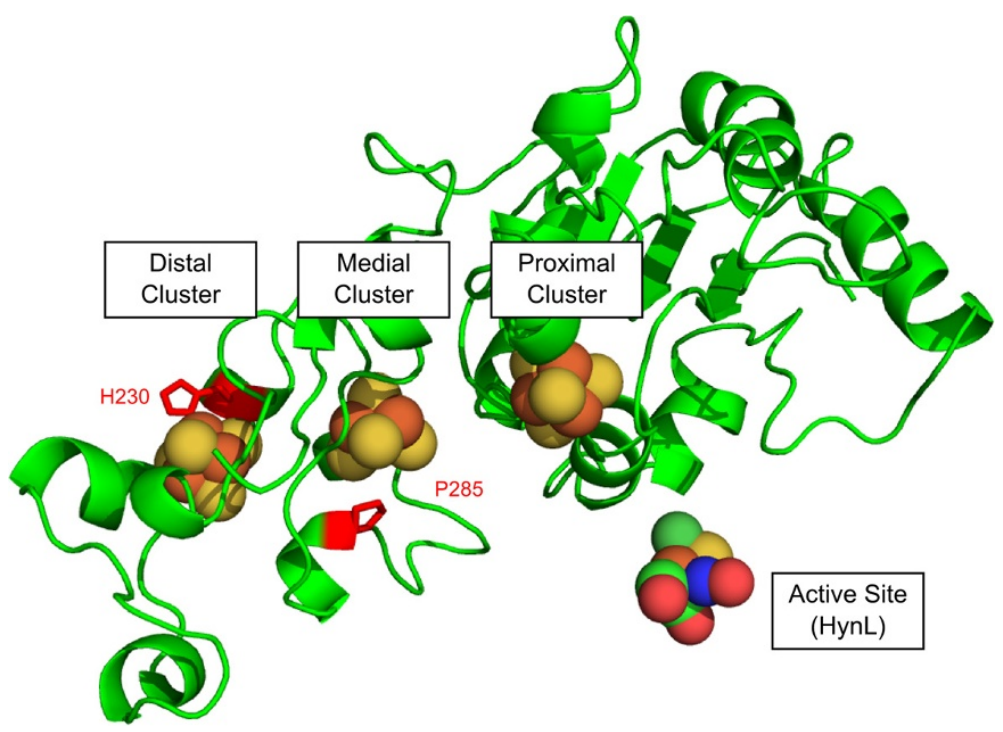

Figure 1 Structural model of the A. macleodii [NiFe] uptake hydrogenase small subunit HynS, highlighting the three Fe-S clusters, and residues His230 and Pro285, which were targeted for substitution to cysteine residues. For reference, the location of the active site is shown, but the rest of the large subunit structure was omitted to simplify viewing of the small subunit. The structural model was generated by Phyre using structure PDB: 1h2a as a model $[27,28]$. 
residue at the distal cluster was changed to cysteine ( $D f$ HynA H184C), hydrogen uptake activity was nearly abolished while evolution activity was only $50 \%$ reduced [23]. We produced enzyme variants featuring substitutions in the A. macleodii hydrogenase at sites homologous to the $D$. fructosovorans uptake hydrogenase substitutions. The variants of the A. macleodii enzyme (HynSL - NCBI accession codes: YP_004425481.1, YP_004425482.1) featuring the corresponding substitutions based on homology (HynS H230C, P285C ${ }^{1}$ ) were created both individually and combined in an effort to find an enzyme with improved hydrogen evolution activity for production of hydrogen in cyanobacteria.

Taking advantage of our previously reported dual-species system, these modified enzymes were first screened in $E$. coli to assess their enzymatic properties. Initial screens with $E$. coli extracts indicated that the doubly-substituted enzyme had the highest hydrogen evolving activity. Purification of the enzyme indicated that this activity was intrinsic to the enzyme variant, and not due to enzyme titer or host factors persistent in crude lysate. Finally, we moved the expression system to the cyanobacterium S. elongatus, which recapitulated the relative activities of the enzymes, validating our general strategy of using $E$. coli as a rapidturnover model system.

\section{Results and discussion}

\section{Heterologous expression for [NiFe] hydrogenase in $\mathrm{E}$. coli}

Heterologous expression of the A. macleodii hydrogenase has been previously reported in both $E$. coli [15] and the cyanobacterium S. elongatus [16]. These reports address general issues of aerobic expression of the A. macleodii hydrogenase, as in both these reports, aerobic handling was used throughout, established as safe in the original report on the enzyme [14]. We have previously determined that the minimal gene set required for optimal activity in $E$. coli consists of the hydrogenase structural genes, hynS and $h y n L$, the six pleiotropic maturation factors, $h y p A B C D E F$, the endoprotease $h y n D$, and two additional genes, hupH and orf2. Additionally, four promoter sites can be used to add additional transcriptional regulation throughout the gene cluster (Figure 2). Expression plasmids used initially consisted of these essential 11 genes driven by a single $\mathrm{P}_{\text {TRC }}$ promoter installed in "promoter site 1" (Figure 2). The $\mathrm{P}_{\mathrm{TRC}}$ promoter functions well in both E. coli and cyanobacteria [33]. Expression plasmids also contain a spectinomycin resistance gene and lacI gene that function in both E. coli and cyanobacteria. Variants containing a $\mathrm{P}_{\mathrm{T} 7}$ promoter or $\mathrm{P}_{\text {TRC }}$ promoters at all four "promoter site"s were also generated. For integration into cyanobacteria, these features are flanked by $1-\mathrm{kb}$ of $S$. elongatus DNA on either side for homologous recombination into neutral site 1 (NS1) [34]. Amino acid substitutions were generated by PCR of the operon using primers which encode for mutations corresponding to the substitutions, followed by assembly with an empty vector. Amino acid substitutions of HynS can be transferred by restriction digest and reassembly process. In this process, the wild type sequence is excised using restriction enzymes whose sites flank the region of interest and the new sequence is installed by reassembly of the vector with PCR products featuring the corresponding nucleic acid sequence mutations (see Methods).

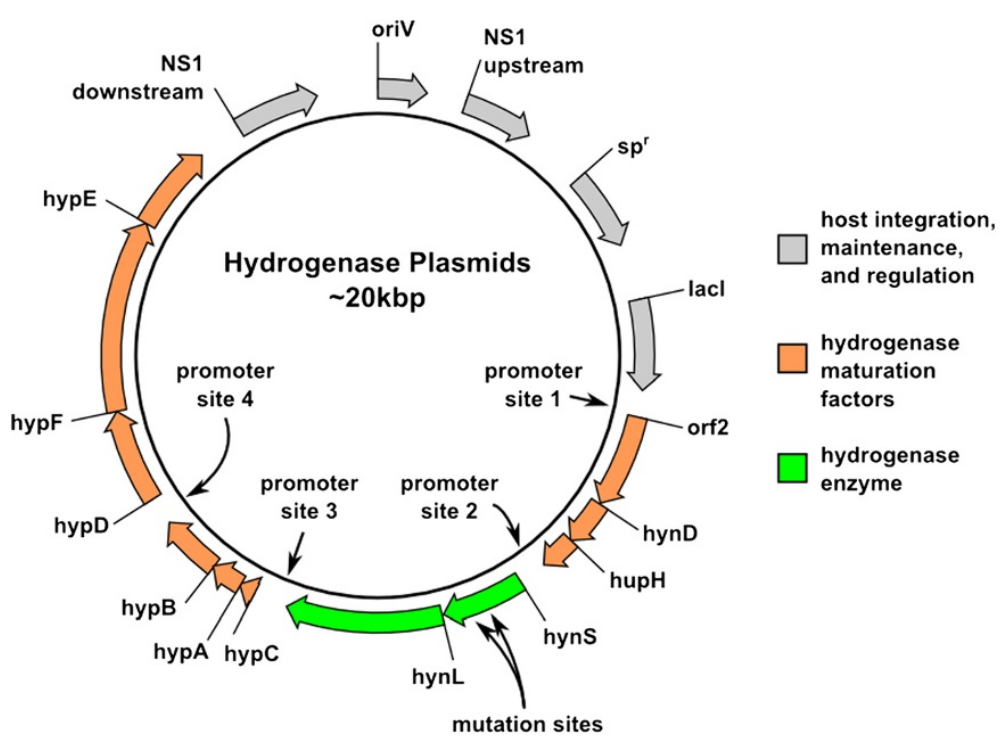

Figure 2 A schematic diagram of the $\boldsymbol{A}$. macleodii hydrogenase expression vectors used in our study. Detailed maps and sequences for all vectors are available in the supplemental information. 


\section{Evolution activity assay}

To test the effect of our substitutions on the hydrogen evolution activity, we performed methyl viologen hydrogen production assays on lysates of $E$. coli cultures expressing the wild type $A$. macleodii hydrogenase (WT), the $A$. macleodii hydrogenase bearing the individual substitutions H230C, P285C, or the doubly-substituted HynS $\mathrm{H} 230 \mathrm{C} / \mathrm{P} 285 \mathrm{C}$. In this assay, electrons are transferred to methyl viologen by oxidation of sodium dithionite, then from methyl viologen to hydrogenase. At the hydrogenase active site, these electrons are recombined with protons to generate molecular hydrogen, which undergoes a phase separation from solution and is detectable by gas chromatographic analysis (GC) of the assay vial headspace. Compared to wild type, lysates containing the singly-substituted enzymes had slightly lower $\mathrm{H}_{2}$ evolution activity. The H230C lysate had approximately half the activity of WT, and the P285C lysate had approximately one third the WT activity (Figure 3A). Lysates containing the H230C/P285C doubly-substituted enzyme, by contrast, exhibited a 34-fold increase in evolution activity compared to WT (Figure 3A).

\section{Uptake activity assay}

To test the effect of our substitutions on hydrogen uptake, we performed a benzyl viologen hydrogen uptake assay on E. coli lysates expressing the wild type $A$. macleodii [NiFe] hydrogenase, and variants featuring the substitutions $\mathrm{H} 230 \mathrm{C}, \mathrm{P} 285 \mathrm{C}$, and $\mathrm{H} 230 \mathrm{C} / \mathrm{P} 285 \mathrm{C}$. In this reaction the hydrogenase catalyzes the dissociation of hydrogen to protons and electrons. The electrons are transferred to benzyl viologen, causing a color change detectable by spectrophotometry at $555 \mathrm{~nm}$. Lysates containing the H230C and P285C enzymes each had slightly increased uptake activity relative to WT; H230C had nearly twice the uptake activity as WT while P285C had slightly less activity than H230C. The hydrogen uptake activity of lysate containing the doublysubstituted enzyme was similar to WT and within the range of experimental error (Figure 3B).

\section{Western blot}

Because our activities are normalized against total protein from crude E. coli lysate, it was necessary to rule out the possibility that differences in hydrogenase expression levels were responsible for the observed differences in activity. Proteins in crude extracts were separated by SDS-PAGE and subjected to western blotting to estimate the relative expression of hydrogenase. Because the primary sequence of HynL is unaffected by the substitutions we made in HynS, we expect the relative intensity of resulting HynL bands to be a good indicator of enzyme titer. Lysates were diluted to $5 \mathrm{mg} \mathrm{mL}^{-1}$ total protein and enzyme activity was measured to ensure activities agreed with those measured previously (Additional file 1: Figure S2). To confirm approximately equivalent loading, a gel slice was stained with Sypro Ruby to illustrate protein content of loaded samples (Figure 4, top). Anti-HynL western blot (Figure 4, bottom) of the lysates shows two bands, presumably corresponding to the immature and the C-terminally processed mature forms of the enzyme subunit [35]. Identically-handled lysate was subjected to brief thermal denaturation $\left(65^{\circ} \mathrm{C}, 10 \mathrm{~min}\right)$ and a second clearing step. Lysate treated in this manner retained nearly all activity (Additional file 1: Figure S2) but lost the larger HynL band in the western blot, lending support to the top band being the unprocessed, immature form of HynL. These western blots suggest that all variants were expressed at approximately equivalent levels and that there were no major defects in expression.

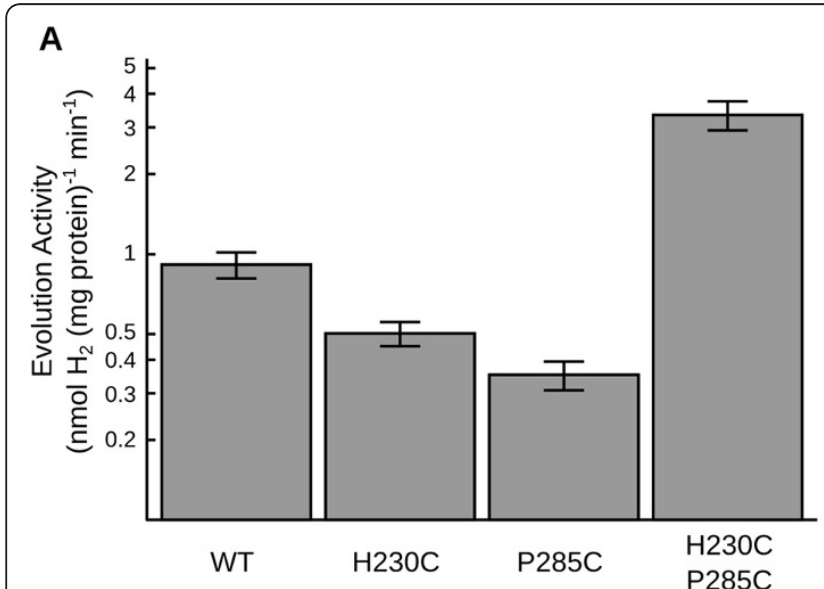

B

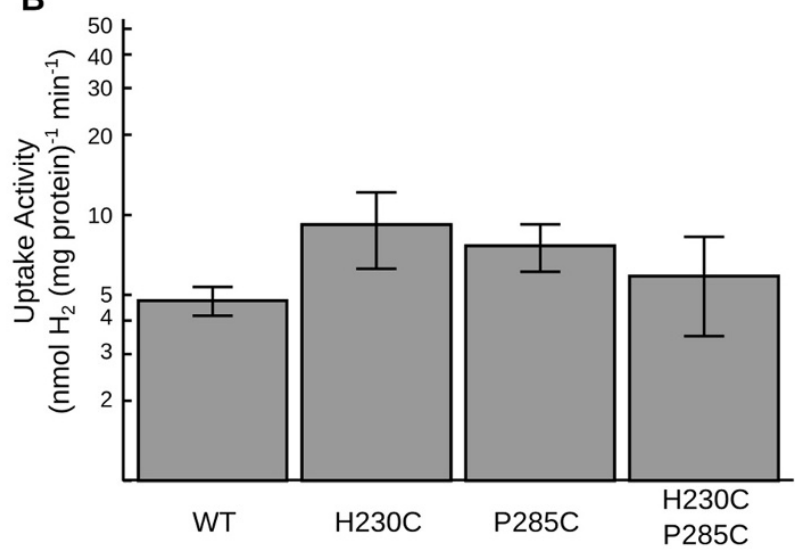

Figure 3 Hydrogen evolution (A) and uptake (B) activities for lysates of $E$. coli strain FTD147 over-expressing wild type $A$. macleodii hydrogenase or substitutions $\mathrm{H230C}, \mathbf{P 2 8 5 C}$, and $\mathbf{H 2 3 0 C / P 2 8 5 C}$. Activities on both graphs are plotted on matching log scales over a 50-fold range to allow comparison of equivalent fold-changes. Error bars represent one standard error of the mean. 


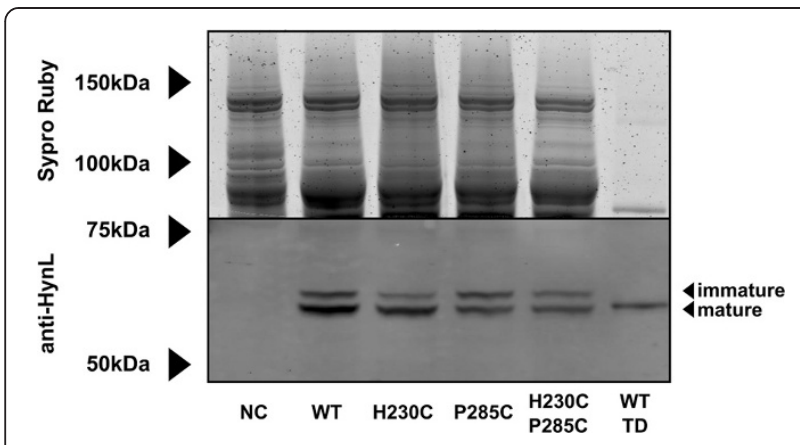

Figure 4 Expression analysis for lysates of $E$. coli strain FTD147 with no hydrogenase construct (NC), over-expressing wild type A. macleodii hydrogenase enzyme (WT), or H230C, P285C, and $\mathrm{H} 230 \mathrm{C} / \mathrm{P} 285 \mathrm{C}$ substitutions, and wild type hydrogenase subjected to thermal denaturation (WT TD). Before loading, proteins were normalized for protein content at $5 \mathrm{mg} \mathrm{mL}^{-1}$. Associated enzyme activites can be found in Figure S1. Top: Sypro-Ruby stain of the gel, indicating approximately equivalent amounts of protein loaded per lane. Bottom: anti-HynL western blot of samples.

\section{Evolution assay on purified enzyme}

To further test if the observed improvement in evolution activity found in the doubly-substituted enzyme is the result of an intrinsic change in enzymatic activity, we generated WT and doubly-substituted enzymes with strep-tags at the C-terminus of HynS. This tag was used in an affinitypurification strategy (Figure 5), and the resulting purified fraction was tested by the hydrogen evolution assay (Additional file 1: Table S1). Some impurities persist in the streptactin-mediated purification; we believe the major contamination in the SDS-PAGE of the strep-tagged protein to be immature small subunit lacking TAT-mediated cleavage, as well as trace amounts of high-molecular weight proteins that persist in the partially purified fractions.

Nonetheless, we found that purification afforded a more than 100-fold improved specific activity for both wild type and doubly-substituted enzymes. Furthermore, after parallel purification the doubly-substituted, strep-tagged enzyme still exhibited three-fold more activity than the wild type, strep-tagged enzyme, consistent with our observations using unpurified $E$. coli lysates in the hydrogen evolution assay (Figure 2A).

\section{Thermal and oxygen tolerance assays}

To test that the doubly-substituted enzyme did not gain improved evolution activity at the expense of other desirable intrinsic properties, we subjected the E. coli lysates to a thermal denaturation assay and an assay testing function in the presence of oxygen. We chose thermal denaturation $\left(85^{\circ} \mathrm{C}\right.$ for $\left.1 \mathrm{~h}\right)$ as a qualitative measure of structural perturbation and hypothesized that the doubly-substituted enzyme would exhibit less thermotolerance than the wild type enzyme due to its modifications. Other treatments were tested; for simplicity we report the harshest treatment we tested that exhibited $>10 \%$ activity. We also tested if the doubly-substituted enzymes would retain function in partial oxygen atmospheres as we had previously observed for the wild-type enzyme. We found that the doubly-substituted enzyme was not less thermotolerant than the WT enzyme within the range of experimental error. Substituting the two residues did not result in a significant defect in the ability to function in the presence of $1 \%$ oxygen (Figure 6).

\section{Relative performance in other bacterial strains}

To test the robustness of our observation that the doublysubstituted enzyme has improved hydrogen evolution activity, we expressed the enzyme with different promoters and in different host species. Plasmid pIY003 expresses the hydrogenase behind a single $\mathrm{P}_{\text {TRC }}$ promoter, with pIY007 as the corresponding plasmid bearing the doubly-substituted enzyme. Plasmid pIY033 expresses the hydrogenase gene cluster behind a single $\mathrm{P}_{\mathrm{T} 7}$ promoter with pIY038 as the corresponding plasmid bearing the doubly-substituted enzyme; pRC41-4 is a plasmid featuring the hydrogenase gene cluster supported by $4 \mathrm{P}_{\mathrm{TRC}}$ promoters with pCM012 as the corresponding plasmid bearing the doubly-substituted enzyme. While pIY003 and pIY007 were tested in the FTD147 strain, the pIY033/pIY038 pair was tested in an $E$. coli strain in which all of its hydrogenases were knocked out (BL21(DE3) $h h y c G \Delta h y a B \Delta h y b C \Delta h y f G$, hereafter referred to as BL21(DE3) $\Delta \mathrm{H}_{4}$ ) [18]. The pRC41-4 and pCM012 plasmids were tested in the cyanobacterial strain S. elongatus PCC 7942 PW416 (DhoxYH:: Em ${ }^{\mathrm{r}}$ ) [16]. Our data indicate that $E$. coli strain FTD147 expressing the doubly-substituted enzyme from pIY007 showed 3.7 \pm 0.6 fold higher activity than the same strain expressing the wild-type enzyme from pIY003; $E$. coli strain BL21 (DE3) $\Delta \mathrm{H}_{4}$ expressing the doubly-substituted enzyme from pIY038 showed $2.3 \pm 0.8$-fold higher activity than the same strain expressing the wild-type enzyme, and an S. elongatus strain expressing the doubly-substituted enzyme from pCM012 showed 5.1 \pm 0.7 -fold higher activity than a strain expressing the wild-type enzyme (Figure 7). Thus, relative to the WT enzyme, the doubly-substituted enzyme consistently shows increased activity -- in both E. coli strains as well as in a cyanobacterial expression system.

By substituting two residues in the small subunit of $A$. macleodii [NiFe] uptake hydrogenase, we have increased its hydrogen evolution activity. To our knowledge this is the first reported instance of this doubly-substituted enzyme analyzed in the context of hydrogen evolution. In the homologous doubly-substituted enzyme from $D$. fructosovorans constructed by Dementin and co-workers [36], activity in the evolving direction was not reported. Comparing the homologous substitutions in A. macleodii and $D$. fructosovorans hydrogenases, we observe qualitatively different structure-activity relationships. Thus, the 


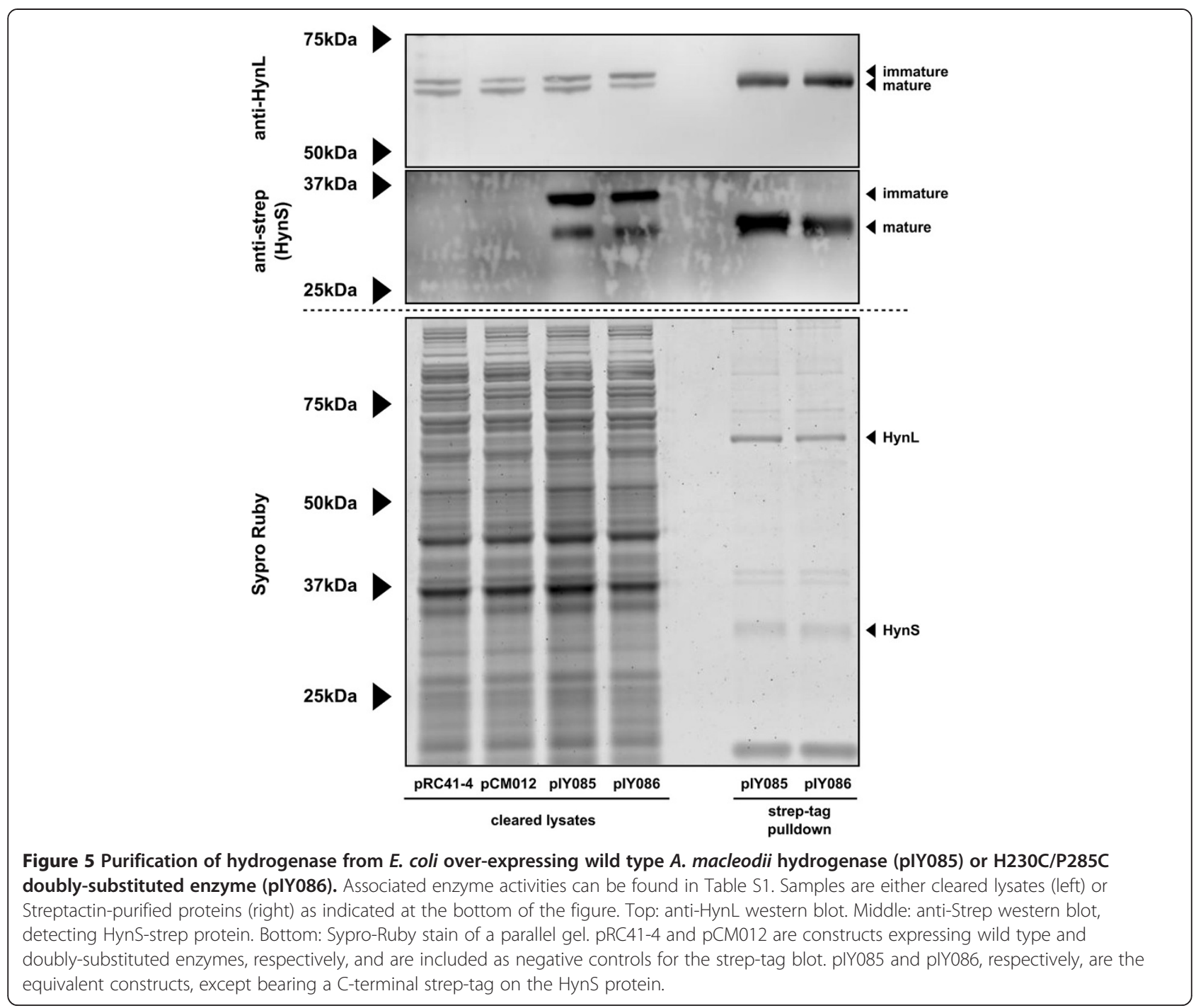

effect of these substitutions may not be generalizable across different members of the enzyme family. Indeed, the inverse experiment, changing a medial $4 \mathrm{Fe}-4 \mathrm{~S}$ cluster to a $3 \mathrm{Fe}-4 \mathrm{~S}$ cluster in the Methanococcus janaschii $\mathrm{F}_{420}$-reducing hydrogenase, resulted in severely impaired uptake, inconsistent with the loose pattern otherwise observed in uptake hydrogenases [31]. As such, others seeking to modify [NiFe] hydrogenase Fe-S cluster ligations should consider testing both single substitutions as well as the double substitution that are homologous to those discussed here when seeking an enzyme suitable for their needs.

The doubly-substituted $A$. macleodii hydrogenase we present here is an attractive launching point for future engineering of hydrogenases redesigned for use in photoautotrophs. The two substitutions have minimal effects on the thermotolerance of the enzyme, as measured by loss of activity after the harsh treatment of $85^{\circ} \mathrm{C}$ for 1 hour. Treatment at lower temperatures or shorter times did not cause appreciable loss of activity in either doubly-substituted or wild type enzymes (data not shown). Our observations suggest that the substitutions have not created severe structural changes, likely allowing further modification of the enzyme without disrupting function. Furthermore, the ability to function in the presence of partial oxygen, a critical property of photoautotrophic hydrogen production systems, appears to be unaffected and may be able to be improved by further modification.

Finally, because $A$. macleodii hydrogenase can be expressed as active enzyme in both $E$. coli [15] and in the photoautotrophic cyanobacterium $S$. elongatus [16], our strategy moving forward is to use expression in $E$. coli as a preliminary model system to rapidly generate and test promising modifications of the enzyme prior to experimentation in S. elongatus, which has a longer turnaround time for genetic manipulations. The $\mathrm{H} 230 \mathrm{C} / \mathrm{P} 285 \mathrm{C}$ enzyme demonstrated the same qualitative performance over the wild type enzyme in extracts prepared from both $S$. elongatus and E. coli, validating 


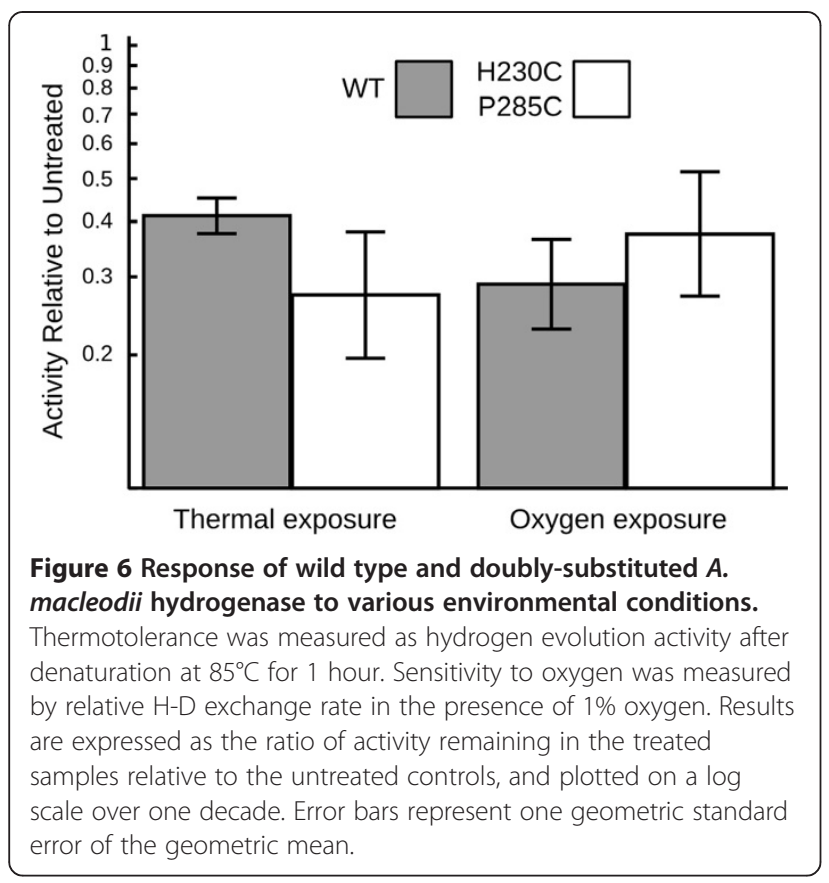

this strategy. Initial attempts at in vivo activity were performed in both host species aerobically and anaerobically, but did not yield measurable hydrogen production. To that end, further modifications we are pursuing to achieve this important result include: improving the oxygen tolerance by engineering a substitution leading to a $4 \mathrm{Fe}-3 \mathrm{~S}$ proximal $\mathrm{Fe}-\mathrm{S}$ cluster that is not natively present in our enzyme [37-40], and connecting the hydrogenase to cyanobacterial electron carriers, such as ferredoxin.

\section{Conclusion}

In summary, we have used an E. coli - cyanobacteria shuttle system to rapidly test candidate modifications of a heterologously-expressed $A$. macleodii [NiFe] hydrogenase in $E$. coli before transferring the most promising constructs to the slower-growing cyanobacteria. As a test of this strategy, we substituted the A. macleodii hydrogenase with modifications that in other systems have been shown to change the ligations of the medial and distal Fe-S clusters. When expressed in E. coli, we found that neither substitution alone improved enzyme activity; however, when combined, both substitutions increased hydrogen evolution activity approximately 4 -fold relative to wild type, while hydrogen uptake remained unchanged. As we did not directly verify that these substitutions resulted in the corresponding alterations to the Fe-S clusters, we cannot completely rule out the possibility of more subtle effects on electron-transfer kinetics in the absence of the expected, new cysteinyl-iron bonds or the possibility that the native enzyme did not feature the expected Fe-S clusters. The effect of this combined modification was recapitulated with the enzymes in enriched form and with the enzymes expressed in cyanobacteria, suggesting that the enzymatic improvement is intrinsic to the enzyme and independent of extrinsic host factors. These results together validate our strategy of piloting hydrogenase modifications in the rapid-turnaround $E$. coli model system for use in cyanobacterial hosts.

\section{Methods}

\section{Molecular biology}

Primers, full plasmid maps and sequences are provided in supplementary materials. Plasmid pIY003 was constructed by PCR-amplification of the hydrogenase operon from $A$. macleodii genomic DNA. Two amplicons spanning the operon were produced where the sequences had overlapping ends with each other and to the plasmid pTRC-NS1 (the backbone plasmid from [16]), which was linearized by double digest with the restriction enzymes BamHI and EcoRI. These three DNA fragments were assembled using Gibson isothermal assembly [41], and transformed into DH10B (Invitrogen) cells by electroporation. After screening clones by PCR, candidate constructs were sequenceverified by Sanger DNA sequencing. Plasmids pIY004, bearing the $\mathrm{H} 230 \mathrm{C}$ substitution and pIY006, bearing the P285C substitution were constructed similarly, except using PCR products generated from primers containing

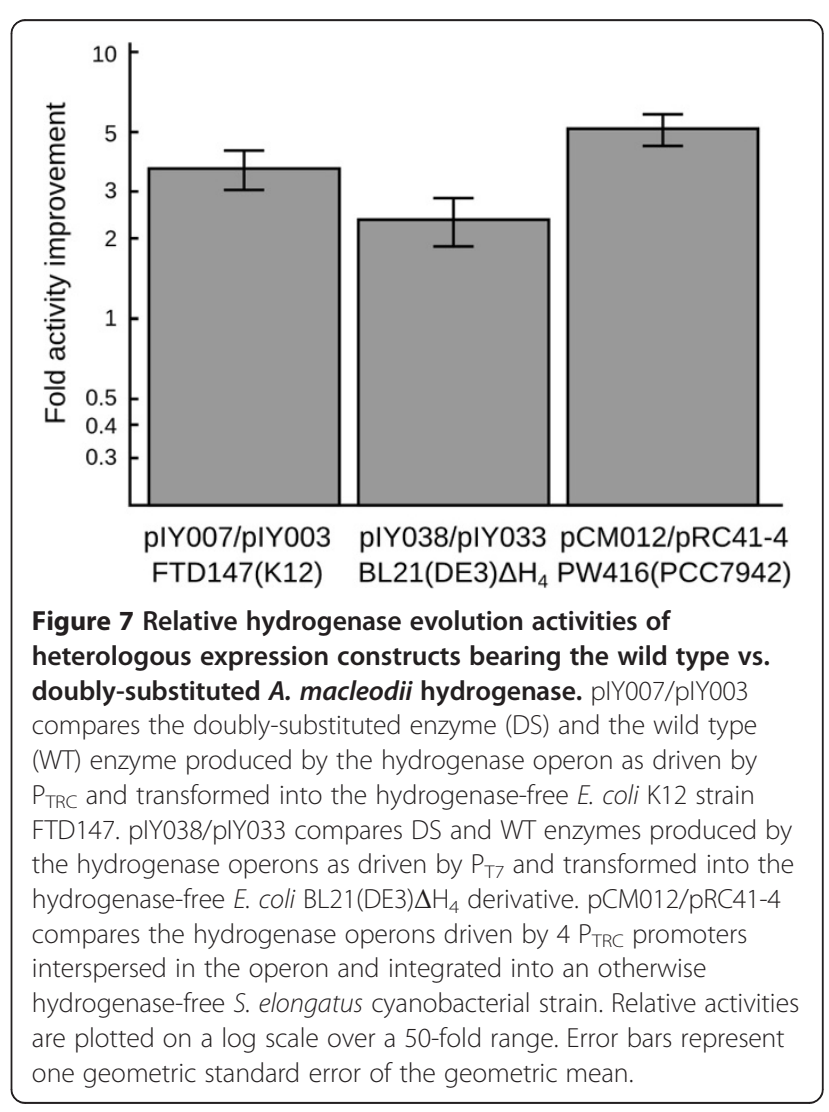


the appropriate DNA nucleotide substitutions; plasmid pIY007, bearing the doubly-substituted enzyme, was constructed by repeating the procedure used to generate pIY006 except using pIY004 plasmid as a template.

Plasmid pIY033 was constructed by moving the hydrogenase operon into a pDEST-23 vector backbone using Gibson isothermal assembly followed by re-amplification of this operon (now including T7 promoter and terminator) and recloning into the pTRC-NS1 plasmid by Gibson isothermal assembly. Successful construction was verified by Sanger sequencing. pIY038 was constructed by PCR amplification of a segment of pIY007 around the hynS ORF followed by Gibson isothermal assembly into plasmid pIY033 which was linearized by double digest with the BamHI and AgeI restriction enzymes. Construction of plasmid pRC41-4 will be discussed elsewhere but the sequence is provided in the Supplemental Information; pCM012 was constructed by PCR amplification of the second half of the hynS ORF from pIY038, followed by Gibson isothermal assembly into pRC41-4 which was linearized by double digest with BamHI and AgeI restriction enzymes. Successful construction of pIY038 and pCM012 was verified by Sanger sequencing initiated with PCR primers used to amplify the modified region. Plasmids pIY085 and pIY086 were constructed similarly to pCM012, with pIY003 and pIY007 as templates, respectively, except using two sequential rounds of amplification with two reverse primers together generating sequence encoding the streptag epitope -RSAWSHPQFEK and the sequence bridging the C-terminal stop codon of hynS and the beginning of hynL up to the AgeI restriction site. Successful construction of pIY085 and pIY086 was verified by Sanger sequencing initiated with the PCR primers used to amplify the modified region.

\section{A. macleodii Hydrogenase Expression in E. coli}

All four plasmids pIY003, pIY004, pIY006, pIY007 were transformed into E. coli strain FTD147 cells, an MC4100 (DE3) strain with the three active $E$ coli hydrogenases removed [42]. Plasmids pIY033 and pIY038 were transformed into BL21(DE3) $\Delta \mathrm{H}_{4}$ cells [18]. To grow E. coli cultures used to prepare extracts, $25 \mathrm{~mL}$ of autoinducer media [43] supplemented with $0.1 \mathrm{mM} \mathrm{NiCl}_{2}$ and $40 \mu \mathrm{g} \mathrm{mL}^{-1}$ spectinomycin was inoculated with $100 \mu \mathrm{L}$ of a starter culture of the appropriate strain. Following inoculation, the expression culture was grown in an unbaffled $250 \mathrm{~mL}$ Erlenmeyer flask, rotating at $200 \mathrm{rpm}$, under ambient room atmosphere at $30^{\circ} \mathrm{C}$ overnight ( $\left.10-14 \mathrm{hrs}\right)$. Under these conditions, the culture grew to a density in the approximate range of $\mathrm{OD}_{600}$ 6-7. Piloting experiments showed that growth in baffled flasks resulted in higher specific activity but was avoided to maintain comparability with previous experiments [15]. This was then harvested by centrifugation; the pellet was separated from the supernatant media and resuspended after addition of $0.8 \mathrm{~mL}$ lysis buffer (10 mM Tris, pH 7; 1 mM DTT; $0.5 \mathrm{mM}$ EDTA). Lysis was performed by sonication on wet ice for one minute using a microprobe sonicator (Branson, Sonifier Model 250) at setting " 4 ", $40 \%$ duty cycle. The lysate was cleared by centrifugation for 10 minutes at $16,800 \mathrm{x} \mathrm{g}$, at $4^{\circ} \mathrm{C}$, and the supernatant was reserved as the "cleared lysate". Protein content in the cleared lysate was measured with Bradford reagent (Bio-Rad) and comparison against a BSA (NEB) standard curve.

\section{A. macleodii Hydrogenase Expression in S. elongatus}

Plasmids pRC41-4 and pCM012 were mobilized into $S$. elongatus PCC 7942 strain PW416 by conjugative transfer from $E$. coli as previously described [16,44]. Genomic integration into neutral site I (NSI) was verified by PCR. Cyanobacterial cultures were grown in $50 \mathrm{~mL}$ Bg11 medium in baffled $250 \mathrm{~mL}$ flasks to an $\mathrm{OD}_{730}$ of approximately 0.5 . The cultures were induced by addition of IPTG to a final concentration of $0.25 \mathrm{mM}$, and additionally $0.5 \mu \mathrm{M} \mathrm{NiCl}$ was supplemented. Cells were grown for an additional $24 \mathrm{~h}$, centrifuged, resuspended in $800 \mu \mathrm{L}$ sonication buffer and sonicated as for E. coli. The lysates were cleared as described above for $E$. coli and the supernatant was reserved as the "cleared lysate". In vitro hydrogenase evolution assays were conducted as described below.

\section{Evolution activity assay}

Cleared lysate $(0.2 \mathrm{~mL})$ was added to $1.7 \mathrm{~mL}$ of methyl viologen assay buffer containing $1.5 \mathrm{~mL}$ deionized water, $0.1 \mathrm{~mL} 40 \mathrm{mg} \mathrm{mL}^{-1}$ methyl viologen (Aldrich), and 0.1 $\mathrm{mL}$ of $500 \mathrm{mM}$ potassium phosphate, pH 7.0 (Gibco). The combined samples in $13 \mathrm{~mL}$ gastight vials was sealed using rubber septa (Aldrich), and sparged under argon (Westair) for 20 minutes to remove oxygen. After sparging, $0.1 \mathrm{~mL}$ of $2 \mathrm{M}$ sodium dithionite (Aldrich) was added by syringe anoxically. The resulting assay solution was a 1:10 dilution of lysate containing $25 \mathrm{mM}$ potassium phosphate, $8 \mathrm{mM}$ methyl viologen, and $100 \mathrm{mM}$ sodium dithionite, $\mathrm{pH} \sim 7.0$. E. coli samples were incubated for 2 hours at $30^{\circ} \mathrm{C}$, followed by gas chromatography (CP-3800, Varian) using a Fused Silica Molsieve 5A column (CP7537, Varian) of $100 \mu \mathrm{L}$ samples taken from the vial headspace. Previous experiments $[13,14]$ and piloting experiments for this experiment demonstrated time-dependent linearity of hydrogen evolution over the course of $\sim 20$ hours, suggesting that enzyme quality is unlikely to have been affected by the reaction conditions, including damage by oxidized dithionite products. S. elongatus samples were prepared similarly but were incubated at $30^{\circ} \mathrm{C}$ overnight before GC analysis. Hydrogen peaks were identified and integrated to quantify hydrogenase yield by comparison to a standard curve prepared with 
pure hydrogen from which a specific activity was calculated based on total protein in the lysate. For plasmids pIY003, pIY004, pIY006, and pIY007, the evolution activity was measured for four biological replicates with three technical replicates for each expression. For plasmids pIY033, pIY038, the evolution activity was measured for three technical replicates, and for pRC41-4, and pCM012, the evolution activity was measured for three biological replicates.

\section{Uptake activity assay}

Cleared lysate $(0.1 \mathrm{~mL})$ was added to $0.9 \mathrm{~mL}$ of benzyl viologen assay buffer containing $0.05 \mathrm{~mL}$ of benzyl viologen $\left(65 \mathrm{mg} \mathrm{mL}^{-1}\right)$ and $0.05 \mathrm{~mL}$ of $0.5 \mathrm{M}$ potassium phosphate buffer, $\mathrm{pH} 7.0$, and $0.8 \mathrm{~mL}$ deionized water. The combined assay sample was prepared in a $2 \mathrm{~mL}$ round-rim disposable UV cuvette (Brandtech) and sealed with a rubber septum (Aldrich), coated with a small amount of vacuum grease (Fisher). The cuvette was sparged for 20 minutes under $10 \%$ hydrogen balanced by nitrogen (Westair). Cuvettes were then transferred to a multichannel spectrophotometer and monitored at 555 $\mathrm{nm}$ at $30^{\circ} \mathrm{C}$. For all four variants, four instances of expression were conducted with four replicates for each instance, such that three of these replicate experiments appeared to have self-consistent progress curves without prejudice to the data processing. Reaction rates were identified by fitting a linear regression to the data between $\mathrm{OD}_{555}=1$ to $\mathrm{OD}_{555}=3$.

\section{Thermal exposure assay}

The assay was conducted as the evolution assay, except cleared lysate was further incubated at $85^{\circ} \mathrm{C}$ for one hour and centrifuged a second time for 10 minutes at 16,800 x g and $4^{\circ} \mathrm{C}$ to clear additional insoluble debris prior to addition to MV buffer. Equivalent volumes of heat-treated and control-treated cleared lysate were added to assay buffer, and total hydrogen evolution activities without normalizing to protein concentration were used for subsequent calculations. Tolerance to thermal exposure was expressed by dividing the mean of three technical replicates of the denatured sample by the mean of three technical replicates of the undenatured sample. This process was subjected to three biological replicates; the geometric mean and geometric standard error are reported.

\section{Oxygen exposure assay}

The H-D exchange assay to test sensitivity to oxygen exposure was performed as follows: cleared lysate $(0.1 \mathrm{~mL})$ was added to $0.8 \mathrm{~mL}$ of mass spectrometry buffer containing $0.05 \mathrm{~mL}$ of $0.5 \mathrm{M}$ potassium phosphate buffer, pH 7.0 (Gibco), and $0.75 \mathrm{~mL}$ deionized water. The samples were placed in $10 \mathrm{~mL}$ scintillation vials, and capped with new rubber septa. Vials were sparged for 10 minutes under pressurized argon. A polyimide-coated fused silica capillary,
ID $50 \mu \mathrm{m}$ OD $220 \mu \mathrm{m}$ (Scientific Instrument Services) adapted for mass spectrometry was then inserted into the vial headspace followed by mass spectrometric monitoring (Omnistar, Pfeiffer). The microcapillary substituted for the standard metal probe supplied with this instrument samples very low quantities of total gas making up an inconsequential amount of total gas volume for the duration of the experiment. Hydrogen (100\%, Westair) was injected to a final concentration of $10 \%$ hydrogen headspace and verified by mass spectrometry. For microaerobic samples, air was injected to create a final $1 \%$ oxygen mixture. Anaerobic samples were incubated for $2 \mathrm{~h}$ at $30^{\circ} \mathrm{C}$ to ensure quantitative enzyme recovery, followed by injection of $0.1 \mathrm{~mL}$ $\mathrm{D}_{2} \mathrm{O}$, followed by monitoring of the developing $\mathrm{m} / \mathrm{z}=3$, until $\mathrm{X}_{\mathrm{HD}}>1000 \mathrm{ppm}$. Microaerobic samples, prepared in parallel, were then injected with $0.1 \mathrm{~mL} \mathrm{D}_{2} \mathrm{O}$ followed by overnight mass spectrometric monitoring at $\mathrm{m} / \mathrm{z}=3$. Oxygen tolerance was measured by filtering spikes from the progress curve, calculating enzyme rate and normalizing the slope of the microaerobic activity against the slope of the anaerobic activity. This process was subjected to three biological replicates; the geometric mean and geometric standard errors are reported.

\section{Protein purification}

Cleared lysate from $E$. coli strains containing plasmid pIY085 and pIY086 was further purified using Streptactin magnetic bead resin (Qiagen). Cleared lysate was prepared as above but in NP buffer ( $50 \mathrm{mM}$ sodium phosphate, $\mathrm{pH}$ 8.0, $300 \mathrm{mM} \mathrm{NaCl}$ ) with $1 \mathrm{mM}$ dithiothreitol and NeutrAvidin (Thermo) at $10 \mu \mathrm{g} \mathrm{mL}{ }^{-1}$ final concentration. Streptactin resin $(100 \mu \mathrm{l})$ was combined with cleared lysate $(400 \mu \mathrm{l})$ and incubated at $4^{\circ} \mathrm{C}$ for $1 \mathrm{~h}$ with end-over-end rotation. The supernatant was removed and the resin was washed three times for five minutes each at room temperature in NP buffer containing $0.05 \%$ sodium dodecyl sulfate (SDS). This was followed by two washes in NP buffer supplemented with $0.01 \%$ Tween 20 as above. Final elution was performed at room temperature in $100 \mu \mathrm{l}$ NP buffer supplemented with $0.01 \%$ Tween 20 and $10 \mathrm{mM}$ biotin.

\section{Western blot}

Lysates were diluted to $5 \mathrm{mg} \mathrm{mL}^{-1}$ total protein. One lysate from an overexpression of WT hydrogenase was further treated by denaturation at $65^{\circ} \mathrm{C}$ for 10 minutes and clearing by centrifugation, at $20,000 \mathrm{x}$ g for 10 minutes. $25 \mu \mathrm{L}$ of $4 \mathrm{x}$ NuPAGE SDS-PAGE loading buffer (Invitrogen) was added to $75 \mu \mathrm{L}$ aliquots, followed by boiling for 10 minutes. These samples were frozen at $-80^{\circ} \mathrm{C}$ and on a subsequent day thawed and $10 \mu \mathrm{L}$ was separated onto a 10\% NuPAGE BisTris gel with the NuPAGE MOPS-SDS running buffer system (Invitrogen). Gel electrophoresis was performed at 150 $\mathrm{V}$ for 5 hours at $4^{\circ} \mathrm{C}$, and the gel was cut separating the gel into two halves at approximately $75 \mathrm{kDa}$. The top gel was 
stained using the Sypro Ruby kit (Invitrogen) as a total protein loading control; the bottom gel was transferred onto nitrocellulose using NuPage transfer buffer (Invitrogen) with $30 \% \mathrm{MeOH}$ for 10 hours at $10 \mathrm{~mA}$ constant current. These samples were then hybridized using rabbit-anti-HynL primary antibody [13] and goat-anti-rabbit-DyLight secondary antibody (Thermo), and imaged using a Typhoon fluorescence scanning imager (GE).

For gels associated with protein purification, samples were prepared as described above, but for cleared lysate, approximately $5 \mu \mathrm{g}$ was loaded per lane and for purified proteins, approximately $0.5 \mu \mathrm{g}$ protein was loaded per lane. Electrophoresis of the gels for western blot analysis of Strep-tagged HynS was only performed for $1 \mathrm{~h}$ at $150 \mathrm{~V}$. Monoclonal antibody to the strep-tag antigen (Qiagen) was used with goat-anti-mouse-DyLight secondary antibody (Thermo) and imaged as above.

\section{Endnotes}

${ }^{1}$ In this paper the amino acid sequence numbers used for $A$. macleodii HynS are relative to the translational start methionine as annotated (NCBI: YP_006975190.1), not the putative starting residue after $\mathrm{N}$-terminal processing by the TAT system, as this site has not been experimentally verified.

\section{Additional file}

Additional file 1: Table S1. Specific activities of enzyme preparations Table S2, Primers used in this study. Table S3, (22pp) Maps and sequences for vectors used in this study. Figure $\mathbf{S 1}$, A representative enzyme activity progress curve.

\section{Abbreviation}

WT: Wild type

\section{Competing interests}

The authors declare that they have no competing interests.

\section{Authors' contributions}

ITY conceived and designed the experiments, created plasmid constructs, collected all data pertaining to E. coli, and wrote the paper. CWM designed and created plasmid constructs, and helped draft the manuscript. TAN designed and created plasmids, and helped draft the manuscript. HOS designed the experiments, participated in its coordination, and helped draft the manuscript. PDW conceived and designed the experiments, collected all data pertaining to $S$. elongatus, performed enzyme purifications, and wrote the paper. All authors read and approved the final manuscript.

\section{Acknowledgements}

We thank Pin-Ching Maness for advice on experimental procedures. We thank Phillip Wright for kindly providing us with the $\mathrm{BL} 21 \Delta \mathrm{H}_{4}$ strain. Funding for all aspects of this work was provided by the U.S. Department of Energy, Fuel Cell Technologies Office (DE-FG36-05GO15027).

Received: 20 February 2013 Accepted: 26 June 2013

Published: 2 July 2013

\section{References}

1. Muellerlanger F, Tzimas E, Kaltschmitt M, Peteves S: Techno-economic assessment of hydrogen production processes for the hydrogen economy for the short and medium term. International Journal of Hydrogen Energy 2007, 32:3797-3810.

2. Beer LL, Boyd ES, Peters JW, Posewitz MC: Engineering algae for biohydrogen and biofuel production. Current opinion in biotechnology 2009, 20:264-271.

3. Angermayr SA, Hellingwerf K, Lindblad P, De Mattos MJ: Energy biotechnology with cyanobacteria. Current opinion in biotechnology 2009, 20:257-263.

4. Ghirardi ML, Dubini A, Yu J, Maness P-C: Photobiological hydrogenproducing systems. Chemical Society reviews 2009, 38:52-61.

5. Vignais PM, Billoud B: Occurrence, classification, and biological function of hydrogenases: an overview. Chemical reviews 2007, 107:4206-4272.

6. Tamagnini P, Leitão E, Oliveira P, Ferreira D, Pinto F, Harris DJ, Heidorn T, Lindblad P: Cyanobacterial hydrogenases: diversity, regulation and applications. FEMS microbiology reviews 2007, 31:692-720.

7. Houchins JP, Burris RH: Occurrence and localization of two distinct hydrogenases in the heterocystous cyanobacterium Anabaena sp. strain 7120. J. Bacteriol 1981, 146:209-214

8. Cournac L, Guedeney G, Peltier G, Vignais PM, De BL: Sustained Photoevolution of Molecular Hydrogen in a Mutant of Synechocystis sp. Strain PCC 6803 Deficient in the Type I NADPH-Dehydrogenase Complex. Journal of Bacteriology 2004, 186:1737-1746.

9. Ghirardi ML, Posewitz MC, Maness P-C, Dubini A, Yu J, Seibert M: Hydrogenases and hydrogen photoproduction in oxygenic photosynthetic organisms. Annual review of plant biology 2007, 58:71-91.

10. Parkin A, Sargent F: The hows and whys of aerobic $\mathrm{H} 2$ metabolism. Current opinion in chemical biology 2012, 16:26-34.

11. Fritsch J, Lenz O, Friedrich B: Structure, function and biosynthesis of $\mathrm{O}(2)$ tolerant hydrogenases. Nature reviews. Microbiology 2013, 11:106-114.

12. McIntosh CL, Germer F, Schulz R, Appel J, Jones AK: The [NiFe]hydrogenase of the cyanobacterium Synechocystis sp. PCC 6803 works bidirectionally with a bias to $\mathrm{H} 2$ production. Journal of the American Chemical Society 2011, 133:11308-11319.

13. Maróti G, Tong Y, Yooseph S, Baden-Tillson H, Smith HO, Kovács KL, Frazier $\mathrm{M}$, Venter JC, Xu Q: Discovery of [NiFe] hydrogenase genes in metagenomic DNA: cloning and heterologous expression in Thiocapsa roseopersicina. Applied and environmental microbiology 2009, 75:5821-5830.

14. Vargas WA, Weyman PD, Tong Y, Smith HO, Xu Q: [NiFe] hydrogenase from Alteromonas macleodii with unusual stability in the presence of oxygen and high temperature. Applied and environmental microbiology 2011, 77:1990-1998

15. Weyman PD, Vargas WA, Chuang R-Y, Chang Y, Smith HO, Xu Q: Heterologous expression of Alteromonas macleodii and Thiocapsa roseopersicina [NiFe] hydrogenases in Escherichia coli. Microbiology (Reading, England) 2011, 157:74-1363

16. Weyman PD, Vargas WA, Tong Y, Yu J, Maness P-C, Smith HO, Xu Q: Heterologous expression of Alteromonas macleodii and Thiocapsa roseopersicina [NiFe] hydrogenases in Synechococcus elongatus. PloS one 2011, 6:e20126.

17. Bock A, King P, Blokesch M, Posewitz M: Maturation of Hydrogenases. Advances in Microbial Physiology 2006, 51:1-225.

18. Wells MA, Mercer J, Mott RA, Pereira-Medrano AG, Burja AM, Radianingtyas $H$, Wright PC: Engineering a non-native hydrogen production pathway into Escherichia coli via a cyanobacterial [NiFe] hydrogenase. Metabolic engineering 2011, 13:445-453.

19. Sun J, Hopkins RC, Jenney FE, McTernan PM, Adams MWW: Heterologous expression and maturation of an NADP-dependent [NiFe]-hydrogenase: a key enzyme in biofuel production. PloS one 2010, 5:e10526.

20. Kim JYH, Jo BH, Cha HJ: Production of biohydrogen by heterologous expression of oxygen-tolerant Hydrogenovibrio marinus [NiFe]hydrogenase in Escherichia coli. Journal of biotechnology 2011, 155:312-319.

21. Lukey MJ, Parkin A, Roessler MM, Murphy BJ, Harmer J, Palmer T, Sargent F, Armstrong FA: How Escherichia coli is equipped to oxidize hydrogen under different redox conditions. The Journal of biological chemistry 2010, 285:3928-3938.

22. Abou Hamdan A, Dementin S, Liebgott P-P, Gutierrez-Sanz O, Richaud P, De Lacey AL, Rousset M, Bertrand P, Cournac L, Léger C: Understanding and tuning the catalytic bias of hydrogenase. Journal of the American Chemical Society 2012, 134:8368-8371.

23. Dementin S, Belle V, Bertrand P, Guigliarelli B, Adryanczyk-Perrier G, De Lacey AL, Fernandez VM, Rousset M, Léger C: Changing the ligation of the 
distal [4Fe4S] cluster in NiFe hydrogenase impairs inter- and intramolecular electron transfers. Journal of the American Chemical Society 2006, 128:5209-5218.

24. Rousset M, Montet Y, Guigliarelli B, Forget N, Asso M, Bertrand P, FontecillaCamps JC, Hatchikian EC: [3Fe-4S] to [4Fe-4S] cluster conversion in Desulfovibrio fructosovorans [NiFe] hydrogenase by site-directed mutagenesis. Proceedings of the National Academy of Sciences of the United States of America 1998, 95:11625-11630.

25. Fontecilla-Camps JC, Volbeda A, Cavazza C, Nicolet Y: Structure/function relationships of [NiFe]- and [FeFe]-hydrogenases. Chemical reviews 2007, 107:4273-4303.

26. Volbeda A, Charon MH, Piras C, Hatchikian EC, Frey M, Fontecilla-Camps JC: Crystal structure of the nickel-iron hydrogenase from Desulfovibrio gigas. Nature 1995, 373:580-587.

27. Higuchi Y, Yagi T, Yasuoka N: Unusual ligand structure in Ni-Fe active center and an additional $\mathrm{Mg}$ site in hydrogenase revealed by high resolution X-ray structure analysis. Structure 1997, 5:1671-1680.

28. Kelley LA, Sternberg MJE: Protein structure prediction on the Web: a case study using the Phyre server. Nature protocols 2009, 4:363-371.

29. Parkin A, Goldet G, Cavazza C, Fontecilla-Camps JC, Armstrong FA: The difference a Se makes? Oxygen-tolerant hydrogen production by the [NiFeSe]-hydrogenase from Desulfomicrobium baculatum. Journal of the American Chemical Society 2008, 130:13410-13416.

30. Garcin E, Vernede X, Hatchikian E, Volbeda A, Frey M, Fontecilla-Camps J: The crystal structure of a reduced [NiFeSe] hydrogenase provides an image of the activated catalytic center. Structure 1999, 7:557-566.

31. Bingemann $\mathrm{R}$, Klein $\mathrm{A}$ : Conversion of the central [4Fe-4S] cluster into a [3Fe-4S] cluster leads to reduced hydrogen-uptake activity of the F420reducing hydrogenase of Methanococcus voltae. European Journal of Biochemistry 2000, 267:6612-6618.

32. Volbeda A, Darnault C, Parkin A, Sargent F, Armstrong FA, Fontecilla-Camps JC: Crystal Structure of the O(2)-Tolerant Membrane-Bound Hydrogenase 1 from Escherichia coli in Complex with Its Cognate Cytochrome b. Structure 2013, 21:184-190.

33. Huang H-H, Camsund D, Lindblad P, Heidorn T: Design and characterization of molecular tools for a Synthetic Biology approach towards developing cyanobacterial biotechnology. Nucleic acids research 2010, 38:2577-2593.

34. Bustos SA, Golden SS: Light-regulated expression of the psbD gene family in Synechococcus sp. strain PCC 7942: evidence for the role of duplicated psbD genes in cyanobacteria. Molecular \& general genetics 1992, 232:221-230.

35. Theodoratou E, Paschos A, Magalon A, Fritsche E, Huber R, Böck A: Nickel serves as a substrate recognition motif for the endopeptidase involved in hydrogenase maturation. European Journal of Biochemistry 2000, 267:1995-1999.

36. Dementin S, Burlat B, Fourmond V, Leroux F, Liebgott P-P, Abou Hamdan A, Léger C, Rousset M, Guigliarelli B, Bertrand P: Rates of intra- and intermolecular electron transfers in hydrogenase deduced from steadystate activity measurements. Journal of the American Chemical Society 2011, 133:10211-10221.

37. Goris T, Wait AF, Saggu M, Fritsch J, Heidary N, Stein M, Zebger I, Lendzian F, Armstrong FA, Friedrich B: others: A unique iron-sulfur cluster is crucial for oxygen tolerance of a [NiFe]-hydrogenase. Nature Chemical Biology 2011, 1:1-9.

38. Pandelia M-E, Nitschke W, Infossi P, Giudici-Orticoni M-T, Bill E, Lubitz W: Characterization of a unique [FeS] cluster in the electron transfer chain of the oxygen tolerant [NiFe] hydrogenase from Aquifex aeolicus. Proceedings of the National Academy of Sciences of the United States of America 2011, 108:6097-6102.

39. Shomura Y, Yoon K-S, Nishihara H, Higuchi Y: Structural basis for a [4Fe-3S] cluster in the oxygen-tolerant membrane-bound [NiFe]-hydrogenase. Nature 2011, 479:253-256.

40. Fritsch J, Scheerer P, Frielingsdorf S, Kroschinsky S, Friedrich B, Lenz O, Spahn CMT: The crystal structure of an oxygen-tolerant hydrogenase uncovers a novel iron-sulphur centre. Nature 2011, 479:249-252.

41. Gibson DG, Young L, Chuang R-Y, Venter JC, Hutchison CA, Smith HO: Enzymatic assembly of DNA molecules up to several hundred kilobases. Nature methods 2009, 6:343-345.

42. Redwood MD, Mikheenko IP, Sargent F, Macaskie LE: Dissecting the roles of Escherichia coli hydrogenases in biohydrogen production. FEMS microbiology letters 2008, 278:48-55.
43. Studier FW: Protein production by auto-induction in high density shaking cultures. Protein expression and purification 2005, 41:207-234.

44. Elhai J, Wolk CP: Conjugal transfer of DNA to cyanobacteria. Methods in enzymology 1988, 167:747-754.

doi:10.1186/1754-1611-7-17

Cite this article as: Yonemoto et al:: Dual organism design cycle reveals small subunit substitutions that improve [NiFe] hydrogenase hydrogen evolution. Journal of Biological Engineering 2013 7:17.

\section{Submit your next manuscript to BioMed Central and take full advantage of:}

- Convenient online submission

- Thorough peer review

- No space constraints or color figure charges

- Immediate publication on acceptance

- Inclusion in PubMed, CAS, Scopus and Google Scholar

- Research which is freely available for redistribution
C Biomed Central 